\title{
Determinação do 1-hidroxipireno em amostras de urina por cromatografia líquida de alta eficiência - estudo dos parâmetros de validação
}

\author{
Patrícia Miranda de Faria*, Henrique Vicente Della Rosa \\ Departamento de Análises Clínicas e Toxicológicas, Faculdade de Ciências Farmacêuticas, \\ Universidade de São Paulo
}

*Correspondência:

P.M. Faria

Faculdade de Ciências Farmacêuticas - USP

Av. Lineu Prestes, $\mathrm{n}^{\circ} 580$

Caixa Postal 66083

05800-960 - São Paulo - SP -Brasil

E-mail: pmfaria@usp.br
Os hidrocarbonetos aromáticos policíclicos (HAPs) correspondem a um grupo de substâncias químicas formadas durante a decomposição térmica de materiais orgânicos e, então, liberadas no meio ambiente. Vários trabalhos têm relatado efeitos adversos produzidos pelos HAPs, sendo muito destes efeitos relacionados ao desenvolvimento de câncer. Segundo a International Agency for Research on Cancer (IARC), seis HAPs são provavelmente carcinogênicos para o homem. Considerando as propriedades toxicológicas e com o intuito de prevenir uma exposição ocupacional a estes compostos, a monitorização biológica por meio da determinação do 1-hidroxipireno urinário é, recomendada. Para tanto, foi necessário validar metodologia para a quantificação do analito proposto. Este trabalho teve como objetivo otimizar as condições de um sistema de cromatografia líquida de alta eficiência (HPLC) e detecção de fluorescência para a análise do 1hidroxipireno urinário e, ainda, validar os parâmetros analiticos para a determinação deste analito em amostras de urina. O método inclui hidrólise enzimática ( $\beta$-glicuronidase/arilsulfatase) com posterior extração em fase sólida - SPE $\left(C_{18}\right)$. A separação cromatográfica foi consumada com uma coluna LC-PAH $(15 \mathrm{~cm} x$ 4,6 mmID x $5 \mu \mathrm{m}$ ), fluxo em fase gradiente (metanol 40 e 100\%) e finalmente detecção seletiva (excitação - $242 \mathrm{~nm}$ e emissão $388 \mathrm{~nm})$. O método demonstrou ser linear $\left(r^{2}=0,999\right)$ na faixa de concentração estudada $(0,2$ a 40,0 $\mathrm{ng} / \mathrm{mL})$. Os limites de detecção e quantificação foram $0,5 \mathrm{ng} / \mathrm{mL}$ e 1,0 $\mathrm{ng} / \mathrm{mL}$, respectivamente. Os resultados de precisão e exatidão foram adequados para a análise $(C V<15 \%)$ e a recuperação do analito manteve-se na faixa de 80 a $120 \%$. Por fim, o método validado foi considerado adequado para a análise do 1-hidroxipireno urinário.
Unitermos

- Análises toxicológicas

- Hidrocarbonetos Aromáticos Policíclicos (HAPs)

- 1-hidroxipireno 


\section{INTRODUÇÃO}

O termo Hidrocarbonetos Aromáticos Policíclicos (HPAs) refere-se a uma classe de compostos orgânicos, que contém dois ou mais anéis aromáticos condensados, constituídos de átomos de carbono $(\mathrm{C})$ e hidrogênio $(\mathrm{H})$. À temperatura ambiente os HAPs são sólidos e as características gerais comuns a esta classe de compostos são: o alto ponto de fusão e ebulição, a baixa pressão de vapor e a pouca solubilidade em água, a qual tende a diminuir com o aumento da massa molecular. Os HAPs são solúveis em muitos solventes orgânicos, altamente lipofílicos e considerados quimicamente inertes (WHO, 1998).

Os HAPs podem ser formados a partir da decomposição térmica de materiais orgânicos, que contenham carbono e hidrogênio. A formação é baseada em dois grandes mecanismos: pirólise ou combustão incompleta e processos de carbonização. Os tipos de HAPs formados parecem depender mais das condições de combustão do que do tipo de material orgânico queimado. A quantidade de HAPs formada sob condições definidas de pirólise depende, no entanto, da temperatura de reação, bem como do material (Bjorseth, 1985).

Desde 1775, quando Sir Percival Pott relatou que limpadores de chaminé na Inglaterra, freqüentemente, desenvolviam alguns tipos de câncer, em particular o escrotal, ouve-se falar dos HAPs. Mais de 150 anos se passaram para que estes compostos fossem reconhecidos como agentes carcinogênicos (Grimmer, 1983; IARC, 1983, 1984, 1985; Bjorseth, 1985; Jongeneelen, 1997; WHO, 1998). Segundo a International Agency for Research on Cancer (IARC), seis HAPs são provavelmente carcinogênicos para o homem: benzo(a)antraceno, benzo(b)fluoranteno, benzo(k)fluoranteno, benzo(a) pireno, dibenzo $(\mathrm{a}, \mathrm{h})$ antraceno e indeno(1,2,3-cd)pireno.

Não obstante o crescente estudo em torno do assunto, inexiste a padronização de procedimentos analíticos para a determinação dos HAPs. Além disto o número de HAPs relatados é desconhecido, fazendo o termo "HAPs Total" incerto. Assim, a fim de reduzir estas incertezas, muitas medidas e estudos são necessários para diversas fontes de emissão. A determinação destas substâncias pode ser realizada através da avaliação ambiental das misturas de HAPs e/ou de um único HAP envolvido. O pireno, um HAP não-carcinogênico, está presente em praticamente todas as misturas de HAPs em concentrações relativamente altas (de 2 a 10\%) e constantes. Há informações de que esta substância está presente como o maior componente do conteúdo total dos HAPs encontrados no ambiente e, em vista de sua abundância, o seu produto de biotransformação, o 1-hidroxipireno (1-OHP), foi seleci- onado para avaliar a exposição a essas substâncias (IARC, 1983; Jongeneelen et al., 1985, 2001; Buchet et al., 1992; Bouchard et al., 1998; Dor et al., 2000).

A difusão destes compostos no ambiente, tanto ocupacional como geral, e a sua característica toxicológica (suspeita e evidência de carcinogenicidade) indicam a grande relevância do estudo do ponto de vista ambiental e biológico com a finalidade de prevenção e promoção da saúde dos trabalhadores. Para tanto, foi necessário validar metodologia específica e sensível para a quantificação precisa e exata do 1-hidroxipireno urinário, já preconizado pela American Conference of Governmental Industrial Hygienists (ACGIH) em 2003 como indicador biológico da exposição aos HAPs. A fim de atingir tal objetivo, este trabalho definiu o estudo dos parâmetros de validação como linearidade, limite de detecção, limite de quantificação, precisão inter e intradia, recuperação e exatidão.

\section{MATERIAL E MÉTODOS}

As amostras de urina destinadas à otimização dos parâmetros para a validação do método analítico para a determinação do 1-OHP foram preparadas a partir de um pool de urina, obtido de alunos de pós-graduação da Faculdade de Ciências Farmacêuticas da Universidade de São Paulo. As amostras foram colhidas, homogeneizadas e adicionadas com solução padrão de 1-hidroxipireno em diferentes concentrações para o preparo da curva de calibração e estudo de linearidade, limite de detecção, limite de quantificação, precisão inter e intradia, recuperação e exatidão.

Neste trabalho foi utilizado um cromatógrafo líquido de alta eficiência Hewlett Packard ${ }^{\circledR}$ (HP), modelo 1100 Series, equipado com desgaseificador a vácuo (G1322A), bomba binária (G1312A), injetor automático (G1313A), compartimento de coluna com termostato (G1316A), detector de arranjo de diodos - DAD (G1315A) e um detector de fluorescência HP 1046A, acoplado a computador modelo Vectra XM, série 4-5/150.

Após a revisão da literatura, optou-se por extrair o analito da urina e identificá-lo segundo Jongeneelen, et al., 1987. A técnica de extração inclui hidrólise enzimática do conjugado com 12,5 de mL $\beta$-glicuronidase/arilsulfatase, seguida de purificação da amostra em um cartucho $\mathrm{C}_{18}$ octadecil sílica (Jongeneelen et al.,1986; Faria, 2003). A separação e a identificação ocorrem por cromatografia líquida de alta eficiência - CLAE com detecção por fluorescência (Jongeneelen et al., 1986, 1987; Buchet et al., 1992; Elovaara et al., 1995; Hatjian et al., 1995; Ovrebo et al., 1995; Bouchard et al., 1996; Moen et al., 1996; Nielsen et al., 1996; Roggi et al., 1997; Lafontaine et al., 2000; Faria, 2003). 
Para verificar a faixa de linearidade do método foram adicionadas, ao pool das amostras de urina, soluçõespadrão de 1-OHP, de modo a obter as seguintes concentrações: 0,$2 ; 0,5 ; 1 ; 2 ; 4 ; 6 ; 8 ; 10 ; 20 ; 40 ; 80 ; 120 ; 160 \mathrm{e}$ $200 \mathrm{ng} / \mathrm{mL}$. A curva de calibração para o 1-OHP foi preparada na faixa de concentração de maior interesse $(0,5$ a $40 \mathrm{ng} / \mathrm{mL}$ ), verificando-se a resposta da curva "área do pico x concentração do 1-OHP”.

Amostras de urina obtidas de seis indivíduos (pool de urina) e amostras de metanol enriquecidas com padrão de 1 -OHP, nas concentrações 0,$5 ; 1 ; 2 ; 5 ; 10 ; 20 ; 30$ e $40 \mathrm{ng} / \mathrm{mL}$ e o branco, foram preparadas em seis replicatas e analisadas. Os dados deste estudo foram utilizados a fim de avaliar a interferência da matriz biológica na análise dentro das condições otimizadas.

O limite de detecção (LD) foi obtido a partir da análise de amostra de urina adicionada de 0,$1 ; 0,2 ; 0,5 \mathrm{e}$ $1,0 \mathrm{ng}$ de $1-\mathrm{OHP} / \mathrm{mL}$ e o limite de quantificação (LQ) foi obtido a partir da análise de amostra adicionada de 1,0 ng de $1-\mathrm{OHP} / \mathrm{mL}$ de urina. Recomenda-se que o LD seja de duas a três vezes superior ao ruído da linha de base e pode ser expresso pela equação: $\mathrm{LD}=\mathrm{DP}$ x 3,3/inclinação da reta e o LQ, definido pela equação: LQ = DP x 10/inclinação da reta, deve ser, no mínimo, cinco vezes superior a qualquer interferência da amostra branco no tempo de retenção do analito. Nas equações citadas o DP corresponde ao desvio-padrão do intercepto com o eixo do y de várias curvas de calibração construídas contendo concentrações do analito próximas ao suposto limite de quantificação. $\mathrm{O}$ pico de resposta do analito no LQ deve ser identificável e reprodutível com precisão de $20 \%$ e exatidão de 80 a 120\% (Chasin et al., 1994, 1998; Brasil, 1999).

Para avaliar a precisão, a recuperação e a exatidão do método, amostras de urina enriquecidas com três con- centrações diferentes: 2,0 (CB), 20,0 (CM) e 30,0 (CA) $\mathrm{ng} / \mathrm{mL}$ de 1-OHP foram preparadas em sextuplicata, segundo o procedimento descrito por Faria (2003), e analisadas no mesmo dia, para o estabelecimento da precisão intradia, e preparadas e analisadas em cinco dias consecutivos, para a precisão interdias. A precisão foi avaliada de acordo com os coeficientes de variação obtidos nas análises. A recuperação do 1-OHP em amostras de urina foi avaliada por comparação da concentração obtida, quando a amostra, contendo o analito, foi submetida ao processo de extração, em relação à concentração obtida quando a amostra, sem a presença do analito, foi submetida ao processo de extração, com adição do padrão antes da análise no HPLC; e a exatidão foi avaliada, por meio da concentração teórica, pela porcentagem de inexatidão ou tendenciosidade (bias) representada pela equação:

Inexatidão $(\%)=\frac{\text { concentração obtida }- \text { concentração teórica }}{\text { concentração teórica }} \times 100$

\section{RESULTADOS}

A otimização de um método precede a validação, já que as condições analíticas, uma vez otimizadas, não devem ser alteradas após a sua validação. Validar é estar com o objetivo voltado para a confiabilidade analítica do laboratório e do método escolhido e otimizado para se obter resultados que se enquadrem às necessidades do problema em questão (Chasin et al., 1994, 1998; Hanks, 1995; Jenke, 1996; Brasil, 1999).

A análise realizada com detecção fluorescente (FLD) mostrou-se específica para o analito proposto, conforme ilustrado na Figura 1 e as condições cromatográ-

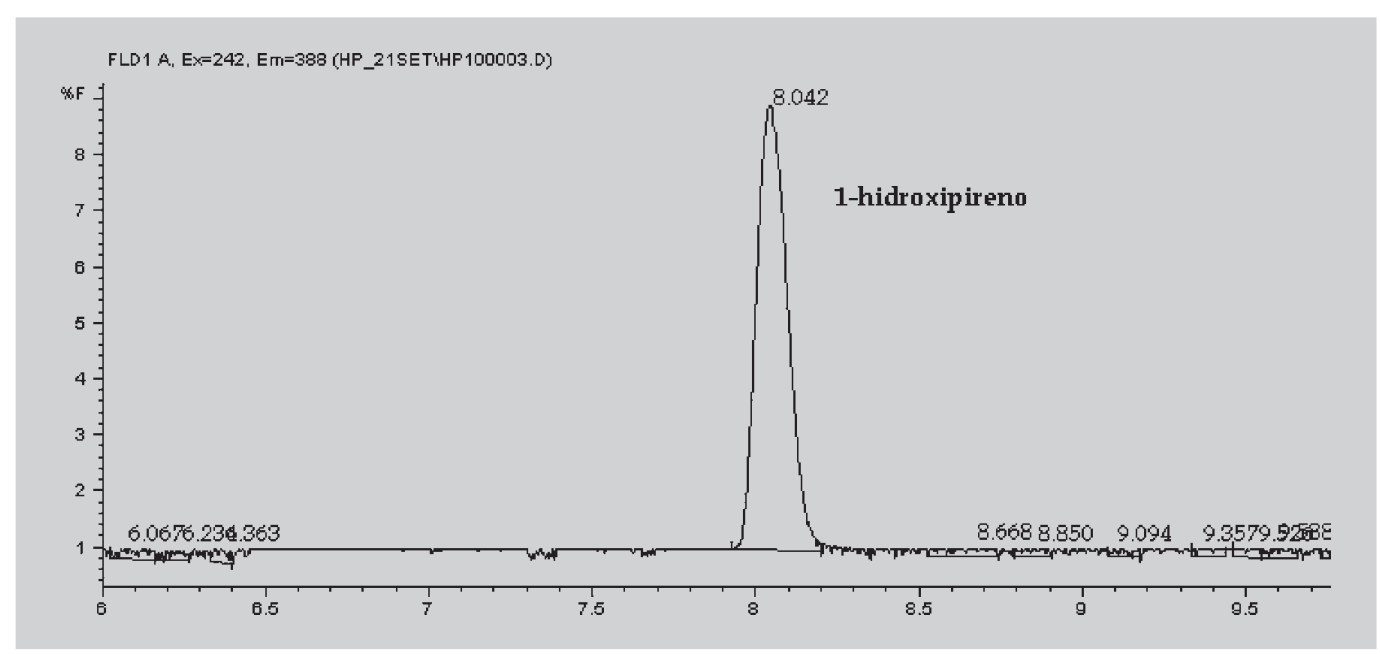

FIGURA 1 - Cromatograma representando o pico do 1-hidroxipireno em amostra adicionada do analito na concentração de 2,5 ng/mL, com tempo de retenção (tr) de 8,042 minutos, fluxo: $0,8 \mathrm{~mL} / \mathrm{min}$ e detector de fluorescência. 
ficas otimizadas para a melhor separação cromatográfica estão expressas na Tabela I.

$\mathrm{O}$ estudo de linearidade do método para 1-OHP foi realizado abrangendo a faixa de 0,2 a $200 \mathrm{ng} / \mathrm{mL}$ (Figura 3 ).

A curva de calibração utilizada para a quantificação do analito em estudo, preparada com um pool de amostras de urina adicionadas com o 1-OHP, apresentou relação linear entre o sinal gerado pelo equipamento e a concentração do 1-OHP na faixa de concentração de 0,5 a 40,0 ng de $1-\mathrm{OHP} / \mathrm{mL}$ de urina, faixa dinâmica de interesse neste estudo. Cada ponto corresponde à média dos valores encontrados na análise das triplicatas. Estes resultados

TABELA I - Condições cromatográficas otimizadas para a análise do 1-OHP urinário

\begin{tabular}{ll}
\hline Condições cromatográficas & 1-OHP \\
\hline Coluna cromatográfica & Supelcosil LC-PAH (15 cm x 4,6 mmID, $5 \mu \mathrm{m})$ \\
Temperatura da coluna & $40{ }^{\circ} \mathrm{C}$ \\
Comprimento de onda no detector de fluorescência & Excitação: $242 \mathrm{~nm}$ Emissão: $388 \mathrm{~nm}$ \\
Eluição gradiente & Metanol $100 \%$ e metanol $40 \%$ \\
Fluxo do eluente & $0,8 \mathrm{~mL} / \mathrm{min}$ \\
\hline
\end{tabular}

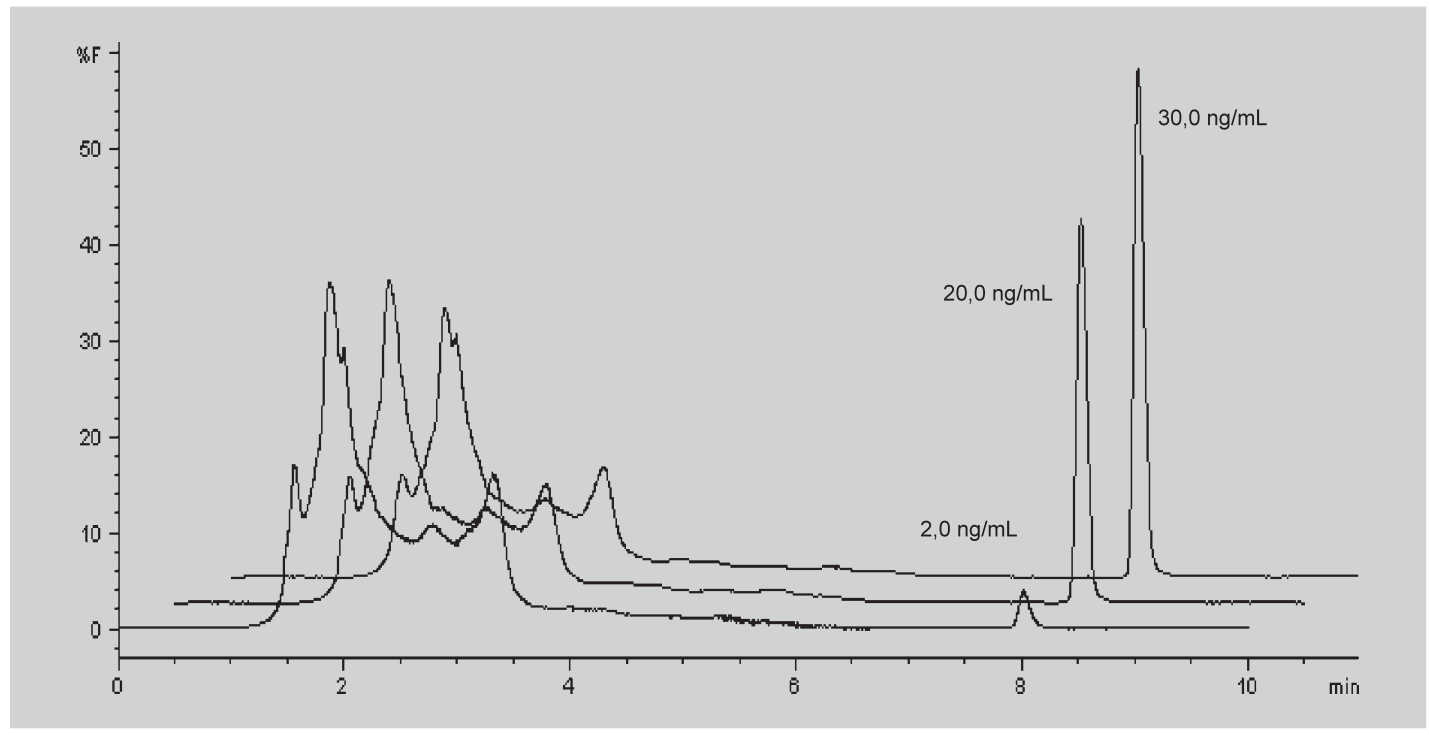

FIGURA 2 - Cromatograma representando o pico do 1-hidroxipireno nas respectivas concentrações, 2,0 - 20,0 e $30,0 \mathrm{ng} / \mathrm{mL}$ com tempo de retenção de 8,042 minutos, fluxo: $0,8 \mathrm{~mL} / \mathrm{min}$ e detector de fluorescência.

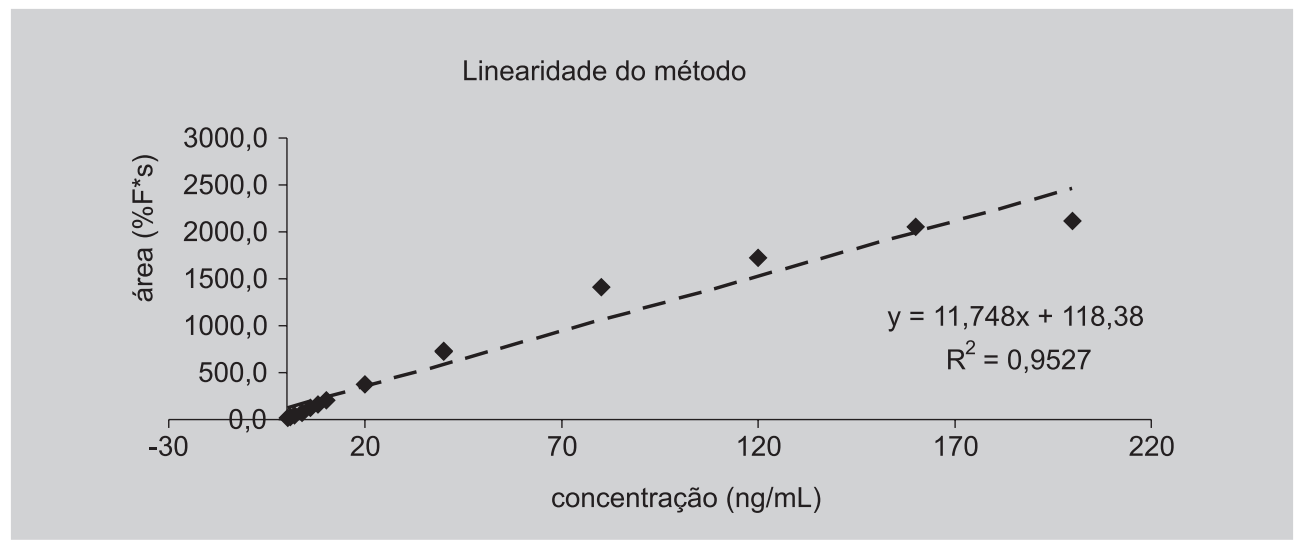

FIGURA 3 - Curva de linearidade do método para a determinação do 1-OHP em amostras de urina, na faixa de 0,2 a $200,0 \mathrm{ng} / \mathrm{mL}$. 
estão demonstrados na Tabela II e Figura 4.

As curvas de calibração em metanol e em amostras de urina podem ser observadas graficamente na Figura 5. As equações de regressão linear são: $y=1,4358 \mathrm{x}-0,7677$ (metanol), com coeficiente de determinação de 0,9986 e $y=15,677 x-11,26$ (urina), com coeficiente de determinação de 0,9897 .

Os resultados para coeficiente angular/inclinação da reta (a) e intercepto (b), da equação da reta $y=a x+b$, estão representados na Tabela III.

TABELA II - Dados obtidos na análise do 1-OHP, em estudo para construção de curva de calibração, através da técnica de CLAE acoplada a detector de fluorescência

\begin{tabular}{cccc}
\hline Concentração $\mathbf{( n g} / \mathbf{m L})$ & Área $\mathbf{( \% F )}$ & Desvio padrão & C.V. \% \\
\hline 0,5 & 7,63 & 0,3620 & 4,79 \\
1,0 & 14,38 & 0,0535 & 0,37 \\
2,0 & 30,46 & 0,6895 & 2,26 \\
5,0 & 69,75 & 0,4989 & 0,72 \\
10,0 & 154,02 & 0,5677 & 0,37 \\
20,0 & 333,06 & 1,5707 & 0,47 \\
30,0 & 412,70 & 1,0440 & 0,25 \\
40,0 & 672,03 & 0,7755 & 0,12 \\
\hline
\end{tabular}

Onde: $\mathrm{CV}=$ coeficiente de variação.

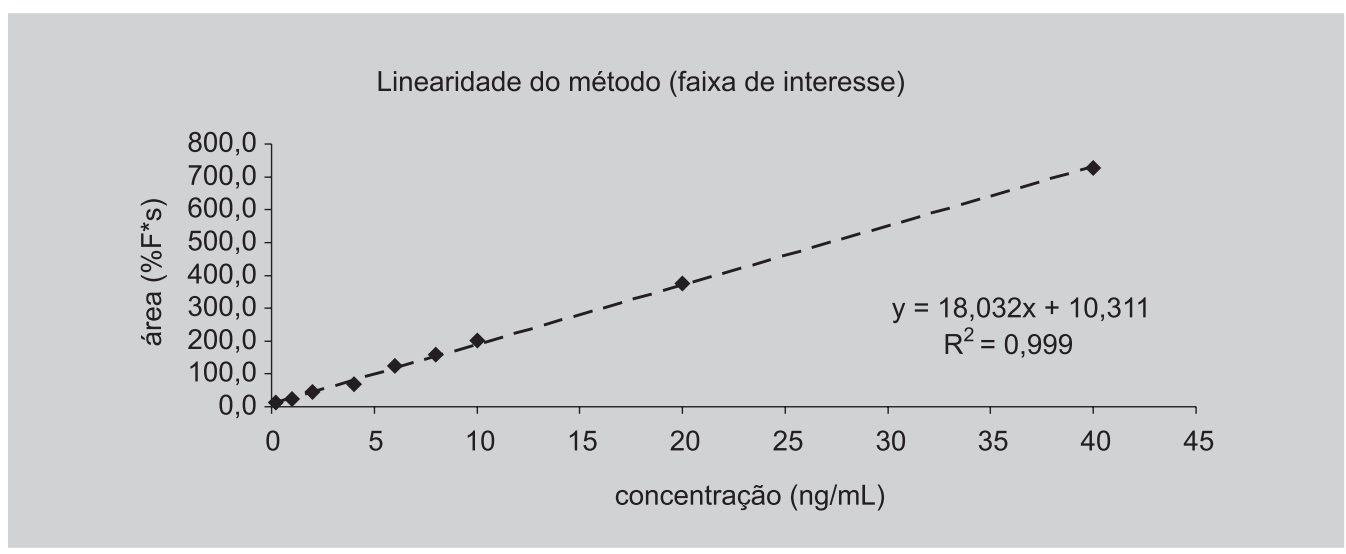

FIGURA 4 - Curva de linearidade do método para a determinação do 1-OHP em amostras de urina, na faixa de 0,2 a $40,0 \mathrm{ng} / \mathrm{mL}$

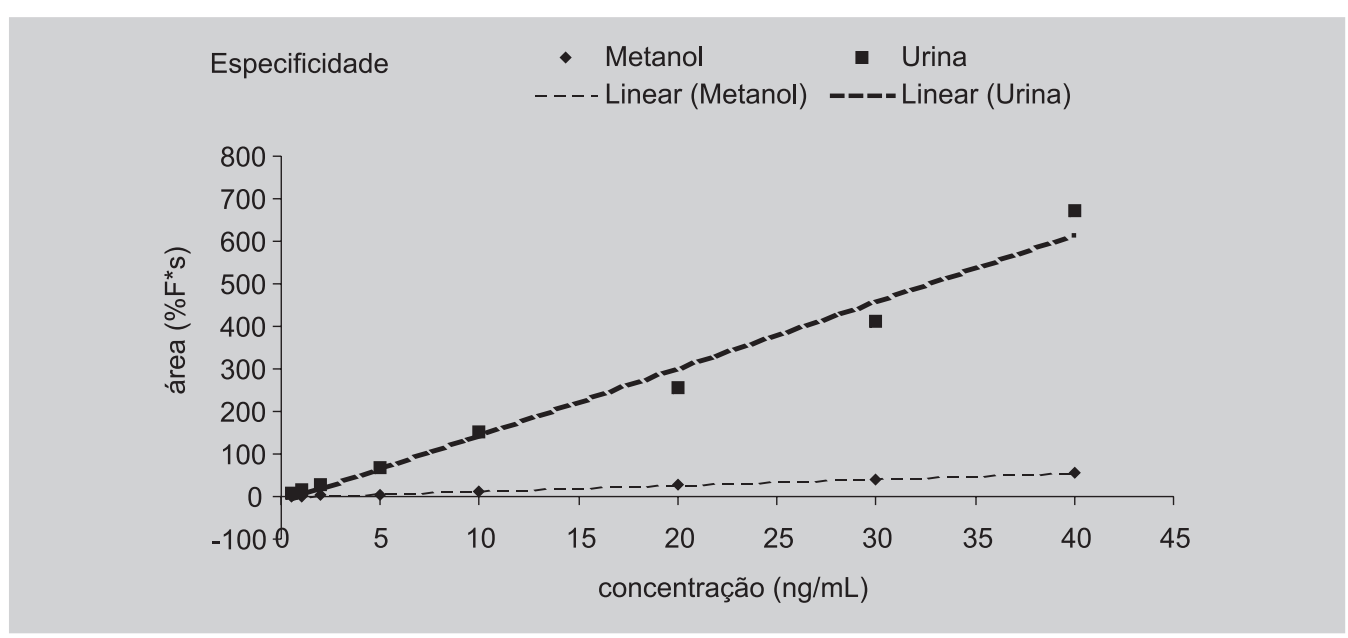

FIGURA 5 - Representação gráfica do estudo de especificidade mostrando as curvas de calibração em metanol ( $\bullet)$ e em urina (ם). 
Obedecendo à definição do LD como sendo a quantidade da amostra que gera uma resposta duas a três vezes maior que o nível do ruído, com coeficiente de variação inferior a $20 \%$, e do LQ como sendo a quantidade da amostra que gera uma resposta cinco vezes maior que o nível do ruído, com precisão de $20 \%$ e exatidão de 80 a 120\% (Collins, Braga, Bonato, 1997; Chasin, et al., 1994, 1998; BRASIL, 1999), os valores de LD e LQ para o 1-

TABELA III - Valores do coeficiente angular/inclinação da reta (a) e do ponto de intersecção no eixo x, denominado intercepto (b), para as curvas de calibração construídas em metanol e urina

\begin{tabular}{llc}
\hline & Metanol & Urina \\
\hline Inclinação (a) & 1,435767 & 15,67707 \\
Intercepto (b) & $-0,76771$ & $-2,82571$ \\
\hline
\end{tabular}

OHP estão descritos nas Tabelas IV e V, e apresentados na Figura 6.

Para demonstrar a confiabilidade do método para a determinação de 1-hidroxipireno em amostras de urina, os resultados, referentes aos parâmetros de precisão, recuperação e exatidão, estão apresentados a seguir.

A precisão intra- e inter-dias (ensaio), obtida por meio de Análise de Variância (ANOVA) e descrita como

TABELA IV - Limite de detecção (LD) e limite de quantificação (LQ) do 1-OHP obtido pelo cálculo do desviopadrão(DP) do intercepto com o eixo do y (b) de várias curvas de calibração construídas contendo concentrações do analito próximas ao suposto limite de quantificação

\begin{tabular}{lccc}
\hline Média \pm DP & Inclinação da reta & LD & LQ \\
\hline $2,13 \pm 2,27$ & 18,655 & 0,40 & 1,22 \\
\hline
\end{tabular}

TABELA V - Limite de detecção (LD) e limite de quantificação (LQ), obtidos obedecendo aos critérios de precisão e exatidão descritos, e utilizados no método para a determinação de 1-OHP em amostras de urina

\begin{tabular}{ccccc}
\hline Conc. $(\mathbf{n g} / \mathbf{m L})$ & Conc. média $(\mathbf{n g} / \mathbf{m L})$ & DP & CV\% & Exatidão (\%) \\
\hline 0,1 & 0,317 & 0,004 & 1,36 & $\mathbf{3 1 6 , 5}$ \\
0,2 & 0,410 & 0,005 & 1,23 & $\mathbf{2 0 4 , 8}$ \\
$0,5 \mathrm{LD}$ & 0,693 & 0,021 & 3,09 & $\mathbf{1 3 8 , 7}$ \\
$1,0 \mathrm{LQ}$ & 1,093 & 0,003 & 0,29 & $\mathbf{1 0 9 , 3}$ \\
\hline
\end{tabular}

$\mathrm{DP}=$ desvio-padrão; $\mathrm{CV}=$ coeficiente de variação; Conc. $=$ concentração

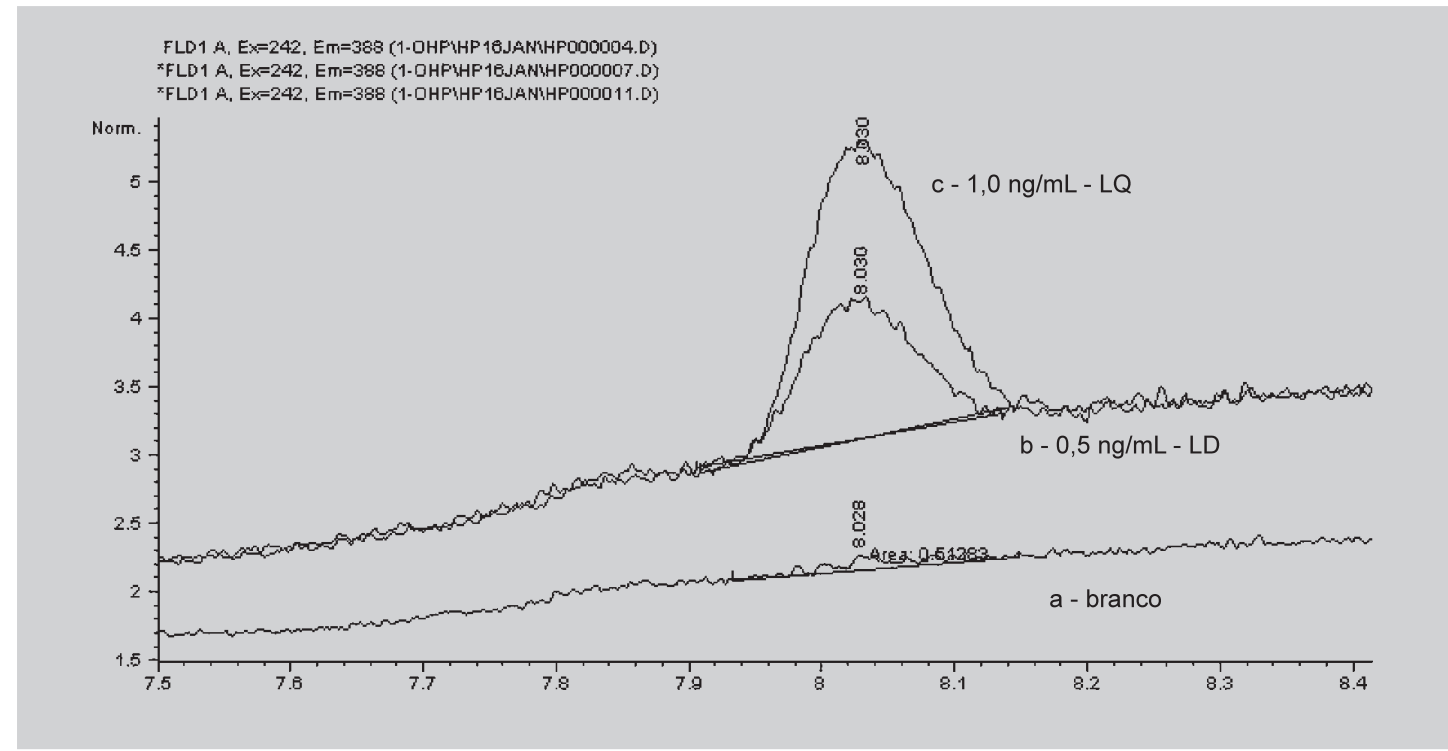

FIGURA 6 - Amostra de urina sem adição de padrão (a), amostra de urina adicionada de $0,5 \mathrm{ng} / \mathrm{mL}$ (b), representando o limite de detecção, e amostra de urina adicionada de $1,0 \mathrm{ng} / \mathrm{mL}$ (c), representando o limite de quantificação. 
coeficiente de variação $(\mathrm{CV})$, com lote de seis amostras de urina adicionadas de 2, 20 e $30 \mathrm{ng} / \mathrm{mL}$, preparadas no mesmo dia e em cinco dias diferentes, respectivamente, está reportada na Tabela VI.

A recuperação mede a eficiência do procedimento de extração de um método analítico dentro de um limite de variação. Porcentagens de recuperação próximas a $100 \%$ são desejáveis, porém, admitem-se valores menores, por exemplo, de 50 a $60 \%$, desde que a recuperação seja precisa.

\section{DISCUSSÃO E CONCLUSÕES}

A procura de indicadores biológicos confiáveis para o estudo da exposição humana aos hidrocarbonetos aromáticos policíclicos (HAPs) estabelece crescente neces- sidade para a realização de diversos estudos e pesquisas. O 1-hidroxipireno urinário tem sido proposto nos estudos de exposição humana aos HAPs e, levando-se em conta que até o presente não se dispõe do Índice Biológico Máximo Permitido (IBMP), o presente estudo foi desenvolvido, com o intuito de colaborar com estas pesquisas.

As análises por CLAE para a separação e identificação dos metabólicos dos HAPs são essencialmente baseadas na separação por fase reversa, aplicada em diversos estudos destes compostos (Jongeneelen et al.,1986, 1987; Buchet et al., 1992; Elovaara et al., 1995; Hatjian et al., 1995; Ovrebo et al., 1995; Bouchard et al, 1996; Moen et al., 1996; Nielsen et al., 1996; Roggi et al., 1997; Lafontaine et al., 2000).

TABELA VI - Coeficiente de variação inter- e intra-dias das concentrações urinárias de 1-hidroxipireno. Análise em sextuplicata e durante cinco dias

\begin{tabular}{cccc}
\hline $\begin{array}{c}\text { Concentração } \\
(\mathbf{n g} / \mathbf{m L})\end{array}$ & $\begin{array}{c}\text { Média das áreas } \\
\left(\mathbf{\%} \mathbf{F}^{*} \mathbf{S}\right)\end{array}$ & $\begin{array}{c}\text { Inter-dia } \mathbf{C V} \\
\mathbf{( \% )}\end{array}$ & $\begin{array}{c}\text { Intra-dia } \mathbf{C V} \\
\mathbf{( \% )}\end{array}$ \\
\hline 2,0 & 28,38 & 7,3 & 4,5 \\
20,0 & 279,87 & 12,38 & 10,9 \\
30,0 & 385,30 & 9,96 & 2,18 \\
\hline
\end{tabular}

Onde: $\mathrm{CV}=$ coeficiente de variação.

TABELA VII - Porcentagem de recuperação (R) do 1-OHP adicionado às amostras de urina nas concentrações de 2,0 20,0 e $30,0 \mathrm{ng} / \mathrm{mL}$

\begin{tabular}{cccccc}
\hline \multirow{2}{*}{$\begin{array}{c}\text { Concentração } \\
\text { (ng/mL) }\end{array}$} & \multicolumn{2}{c}{ Adição antes da extração } & \multicolumn{2}{c}{ Adição após extração } & R (\%) \\
Média \pm DP $(\mathbf{n g} / \mathbf{m l})$ & $\mathbf{C V \%}$ & Média \pm DP $(\mathbf{n g} / \mathbf{m l})$ & $\mathbf{C V \%}$ & \\
\hline 2,0 & $2,00 \pm 0,063$ & 3,16 & $1,88 \pm 0,027$ & 1,45 & 106,38 \\
20,0 & $23,37 \pm 0,135$ & 0,58 & $20,41 \pm 0,962$ & 4,72 & 114,50 \\
30,0 & $34,33 \pm 0,150$ & 0,44 & $29,79 \pm 2,406$ & 8,09 & 115,43 \\
\hline
\end{tabular}

$\mathrm{DP}=$ desvio-padrão; $\mathrm{CV}=$ coeficiente de variação; concentração em ng/mL.

TABELA VIII - Estudo de exatidão com amostras de urina adicionadas de padrão de 1-OHP

\begin{tabular}{cccc}
\hline $\begin{array}{c}\text { Concentração } \\
\text { teórica }(\mathbf{n g} / \mathbf{m L})\end{array}$ & $\begin{array}{c}\text { Concentração } \\
\text { média obtida }(\mathbf{n g} / \mathbf{m L})\end{array}$ & $\mathbf{C V \%}$ & Inexatidão (\%) \\
\hline 2,0 & 2,04 & 1,81 & $\pm 2,24$ \\
20,0 & 20,27 & 7,66 & $\pm 1,33$ \\
30,0 & 27,37 & 1,27 & $\pm 8,78$ \\
\hline
\end{tabular}

$\mathrm{CV}=$ coeficiente de variação 
A análise por cromatografia líquida em fase reversa do 1-hidroxipireno (metabólico hidroxilado dos HAPs) foi satisfatória, como pode ser observado nas Figuras 1 e 2, com cromatogramas em alta resolução e baixo nível de compostos interferentes na faixa do analíto em estudo.

Considerando a viabilidade na determinação do 1-OHP conforme relatado na literatura cientifica é necessário que a validação da metodologia, a ser adotada, seja realizada.

No estudo da linearidade do método não foi possível obter uma relação linear entre a concentração do 1$\mathrm{OHP}$, na faixa de 0,2 a $200 \mathrm{ng}$ de $1-\mathrm{OHP} / \mathrm{mL}$ de urina, e a resposta do detector, pois o coeficiente de determinação $\left(\mathrm{r}^{2}\right)$ de 0,9527 foi insatisfatório (Figura 3) (Chasin et al., 1994). Todavia, os dados obtidos pelo estudo de linearidade na faixa de interesse do método $(0,2$ a 40,0 ng $1-\mathrm{OHP} / \mathrm{mL}$ urina) demonstraram uma resposta linear entre a concentração do analito e a intensidade do sinal gerado por porcentagem $(\%)$ de fluorescência, satisfatória $\left(\mathrm{r}^{2}=0,999\right)$, conforme preconizado por Chasin et al. $(1994,1998)$ (Figura 4). O estudo de linearidade foi primeiramente realizado desconhecendo-se as reais concentrações do 1-OHP urinário durante uma exposição ocupacional.

De acordo com alguns autores, a sensibilidade é definida como a inclinação da curva de calibração que possibilite a medida em qualquer ponto (Miller, Miller, 1988; Angerer et al., 1992; Chasin et al., 1998; Leite, 1998). Quanto maior for a inclinação da curva de calibração maior será a sensibilidade e especificidade do método. No presente estudo, a curva de calibração, preparada com um pool de amostras de urina, apresentou inclinação de 15,68 e a curva de calibração, preparada em metanol, apresentou inclinação de 1,44 (Figura 5 e Tabela III). A análise destes valores de inclinação demonstra que o método é mais seletivo e sensível quando a quantificação do analito é realizada com uma curva de calibração preparada com amostras de urina.

Os limites de detecção e quantificação foram adequados para a finalidade proposta. Obedecendo ao critério preconizado pela ANVISA (Brasil, 1999) os resultados adotados neste trabalho foram de $0,5 \mathrm{ng} / \mathrm{mL}$ para detecção e 1,0 ng/mL para uma quantificação precisa e exata do 1OHP urinário.

A precisão do método é o parâmetro que avalia a proximidade entre as várias medidas efetuadas em uma mesma amostra. Este parâmetro deve ser determinado em um mesmo dia (precisão intra-dia) e em dias diferentes (precisão inter-dias). A medida de precisão pode ser expressa através do cálculo do desvio padrão e do coeficiente de variação (CV\%), não se admitindo valores superio- res a $15 \%$, obtidos em condições determinadas de repetibilidade e/ou reprodutibilidade (Jenke, 1996; Chasin et al, 1998; FDA, 1998; Brasil, 1999). Recomenda-se, no mínimo, a análise de três concentrações (baixa, média e alta) dentro da faixa de limite esperado, realizando-se, pelo menos, cinco réplicas (Brasil, 1999). Neste trabalho o estudo de precisão foi realizado em sextuplicata, o que permitiu o tratamento estatístico das amostras em um intervalo de confiança de $95 \%$ de probabilidade na tabela $\mathrm{t}$ de Student (Chasin et al., 1998).

A imprecisão entre dias expressada como coeficiente de variação $(\mathrm{CV})$ foi relatada na literatura em diversos trabalhos como 13,3\%, 5,5\% e 5,9\% nas concentrações de 6, 3 e $1 \mathrm{ng} / \mathrm{mL}$, respectivamente (Roggi, 1997; Siwinska, 1998).

De acordo com o preconizado e adotado neste trabalho, os resultados de precisão obtidos foram considerados satisfatórios e adequados $(\mathrm{CV}<15 \%)$. Os resultados, mesmo que de maneira aproximada, estão de acordo com os relatados na literatura para o analito em estudo. O estudo de recuperação demonstrou ser adequado, com resultados dentro da faixa de aceitação de 80 a 120\% (Chasin et al., 1994, 1998; Brasil, 1999), conforme descritos na Tabela VII e os resultados obtidos no estudo de exatidão e reportados na Tabela VIII estão de acordo com o preconizado, com uma porcentagem (\%) de inexatidão menor que os 15\% sugeridos (Chasin et al., 1998).

A validação do método para a determinação do 1OHP em amostras de urina, sugerido por Jongeneelen (1986), foi satisfatória atendendo os objetivos deste trabalho.

\section{ABSTRACT}

\section{Determination of 1-hydroxypyrene in human urine by high-performance liquid chromatography - study of validation parameters}

Polycyclic aromatic hydrocarbons (PAHs) constitute a group of chemical substances formed during thermal degradation of organic materials released in the environment. At present, several hundreds of papers reporting adverse effects produced by PAHs are mainly related to cancer development. According to International Agency for Research on Cancer (IARC), six PAHs are probably human carcinogenic. Considering the toxicological properties and with the aim to prevent occupational exposures to these substances, a biological monitoring of urinary 1-hydroxypyrene is recommended. The first step to achieve this goal is to validate a methodology for urinary 1-OHP quantification. This work 
optimized the conditions of a high performance liquid chromatography system (HPLC) with fluorescence detection and validated the analytical parameters for urine 1-OHP determinations. The method includes an enzymatic hydrolysis (b-glucuronidase/arylsulfatase) followed by a solid phase extraction - SPE $\left(C_{18}\right)$. In addition a chromatographic separation was accomplished using a Supelcosil ${ }^{T M}$ LC-PAH $(15 \mathrm{~cm} \times 4.6 \mathrm{mmID} \times 5 \mu \mathrm{m})$ with a gradient phase (methanol 40 and $100 \%$ ) and finally a selective detection (excitation - $242 \mathrm{~nm}$ and emission$388 \mathrm{~nm})$. The method showed linearity $\left(r^{2}=0.999\right)$ with the studied range of concentration ( 0.2 to $40.0 \mathrm{ng} / \mathrm{mL}$ ). Detection and quantification limit were $0.5 \mathrm{ng} / \mathrm{mL}$ and $1.0 \mathrm{ng} / \mathrm{mL}$, respectively. Relative standard deviation of the method was good $(C V<15 \%)$, as also was the reproducibility (recovery maintained between 80 and $120 \%$ ). In conclusion, this validated method can be considered useful for the urinary 1-OHP analyses.

UNITERMS: Toxicological analyses. Polycyclic aromatic hydrocarbons (PAHs). 1-Hydroxypyrene. Biological monitoring.

\section{AGRADECIMENTOS}

À FAPESP, pela concessão da bolsa de Doutorado (processo 00/05556-7).

\section{REFERÊNCIAS BIBLIOGRÁFICAS}

AMERICAN CONFERENCE OF GOVERNMENTAL INDUSTRIAL HYGIENISTS. 2003 TLVS and BEIs based on the documentation for threshold limit values for chemical substances and physical agents and biological exposure indices. Cincinnati: ACGIH, 2003. 219p.

ANGERER, J.; HORSCH, B. Determination of aromatic hydrocarbons and their metabolits in human blood and urine. J. Chromatogr., Amsterdam, v.580, p.229255,1992 .

BJORSETH, A.; RAMDAHL, T. Handbook of polycyclic aromatic hydrocarbons. New York: Marcel Dekker, 1985. v.2, 416p. (Emission Sources and Recent Progress in Analytical Chemistry).

BOUCHARD, M.; VIAU, C. Urinary and biliary excretion kinetics of 1-hydroxypyrene following intravenous and oral administration of pyrene in rats. Toxicology, Amsterdam, v.127, p.69-84, 1998.
BRASIL. Ministério da Saúde. Agência Nacional de Vigilância Sanitária. Medicamentos. Legislação. Resoluções. Resolução n.391, de 09 de agosto de 1999. Anexo III: Guia para validação de métodos analíticos. Disponível em: $<$ http://www.anvisa.gov.br/legis/resol/ 391_99.htm>. Acesso em: 14 jul. 2001.

BUCHET, J.P.; GENNART, J.P.; CALDERON, F.M.; DELAVIGNETTE, J.P.; CUPERS, L.; LAUWERYS, R. Evaluation of exposure to polycyclic aromatic hydrocarbons in a coke production and a graphite electrode manufacturing plant: assessment of urinary excretion of 1-hydroxypyrene as a biological indicator of exposure. Br. J. Ind. Med., London, v.49, p.761-768, 1992.

CHASIN, A.A.M.; CHASIN, M.; SALVADORI, M.C. Validação de métodos cromatográficos em análises toxicológicas. Rev. Farm. Bioq. Univ. São Paulo, São Paulo, v.30, p.49-53, 1994.

CHASIN, A.A.M.; NASCIMENTO, E.S.; RIBEIRONETO, L.M.; SIQUEIRA, M.E.P.B.; ANDRAUS, M.H.; SALVADORI, M.C.; FERNÍCOLA, N.A.G.; GORNI, R.; SALCEDO, S. Validação de métodos em análises toxicológicas: uma abordagem geral. Rev. Bras. Toxicol., São Paulo, v.11, p.1-6, 1998.

COLLINS, C.H.; BRAGA, G.L.; BONATO, P.S. Introdução a métodos cromatográficos. Campinas: Editora da UNICAMP, 1997. 279p.

DOR, F.; HAGUENOER, J.M.; ZMIROU, D.; EMPEREURBISSONNET, P.; JONGENEELEN, F.J.; NEDELLEC, V.; PERSON, A.; FERGUSON, C.C.; DAB, W. Urinary 1-hydroxypyrene as a biomarker of polycyclic aromatic hydrocarbons exposure of workers on a contaminated site: influence of exposure conditions. J. Occup. Environ. Med., London, v.42, p.391-397, 2000.

ELOVAARA, E.; HEIKKILÄ, P.; PYY, L.; MUTANEN, P.; RIIHIMÄKI, V. Significance of dermal and respiratory uptake in creosote workers: exposure to polycyclic aromatic hydrocarbons and urinary excretion of 1hydroxypyrene. J. Occup. Environ. Med., Baltimore, v.52, p.196-203, 1995.

FARIA, P.M. Avaliação da exposição ocupacional aos hidrocarbonetos aromáticos policíclicos. São Paulo, 2003. 162p. [Tese de Doutorado - Faculdade de Ciências Farmacêuticas - Universidade de São Paulo]. 
FDA. U.S. Food and Drug Administration. Guidance for industry - Bioanalytical method validation. Disponível em: <http://www.fda.gov/cder/guidance/4252fnl.htm>. Acesso em: 20 jan. 2001

GRIMMER, G.; JACOB, J.; DETTBARN, G.; NAUJACK, K.W. Determination of urinary metabolites of polycyclic aromatic hydrocarbons (PAH) for the risk assessment of PAH-exposed workers. Int. Arch. Occup. Environ. Health, v.69, p.231-239, 1997.

HATJIAN, B.A.; EDWARDS, J.W.; HARRISON, J.; WILLIAMS, F.M.; BLAIN, P.G. Ambient, biological and biological effect monitoring of exposure to polycyclic aromatic hydrocarbons (PAHs). Toxicol. Lett., v.77, p.271-279, 1995.

INTERNATIONAL AGENCY FOR RESEARCH ON CANCER. Polynuclear aromatic compounds: Part 1. Chemical environmental and experimental data. Lyon: IARC, 1983. 477 p. (IARC Monograph, n.32).

\section{INTERNATIONAL AGENCY FOR RESEARCH ON} CANCER. Polynuclear aromatic compounds: Part 3. Industrial exposure in aluminum production, coal gasification, coke production, and iron and steel founding. Lyon: IARC, 1984a. 219 p. (IARC Monograph, n.34).

INTERNATIONAL AGENCY FOR RESEARCH ON CANCER. Polynuclear aromatic compounds: Part 4. Bitumens, coal tars and derived products, shale-oils and soots. Lyon: IARC, 1984b. 271 p. (IARC Monograph, n.35), (Working Group on the Evaluation of the Carcinogenic Risk of Chemicals to Humans. Meeting, Lyon, 1985).

JENKE, D.R. Chromatographic method validation: a review of currents practices and procedures. General concepts and guidelines. J. Liq. Chromatogr. Relat. Technol., New York, v.19, p.719-736, 1996.

JONGENEELEN, F.J.; ANZION, R.B.M.; LEIJDEKKERS, Ch.M.; BOS, R.P.; HENDERSON, P.Th. 1Hydroxypyrene in human urine after exposure to coal tar and a coal tar derived product. Int. Arch. Occup. Environ. Health, v.57, p.47-55, 1985.
JONGENEELEN, F.J.; ANZION, R.B.M.; HENDERSON, P.Th. Determination of hydroxylated metabolites of polycyclic aromatic hydrocarbons in urine. $J$. Chromatogr., v.413, p.227-232, 1987.

JONGENEELEN, FJ. Methods for routine biological monitoring of carcinogenic PAH-mixtures. Sci. Total Environ., Amsterdam, v.199,p.141-149, 1997.

JONGENEELEN, FJ. Benchmark guideline for urinary 1hydroxypyrene as biomarker of occupational exposure to polycyclic aromatic hydrocarbons. Ann. Occup. Hyg., Amsterdam, v.45, p.3-13, 2001.

LAFONTAINE, M.; PAYAN, J.P.; DELSAUT, P.; MORELE, Y. Polycyclic aromatic hydrocarbon exposure in an artificial shooting target factory: assessment of 1hydroxypyrene urinary excretion as a biological indicator of exposure. Ann. Occup. Hyg., v.44, p.89-100, 2000.

MILLER, J.C.; MILLER, J.N. Statistics for analytical chemistry. 2.ed. Chischester: Ellis Horwood, 1988.117p. (Ellis Horwood series in analytical chemistry).

MOEN, B.E.; NILSSON, R.; NORDLINDER, R.; OVREBf, S.; BLEIE, K.; SKORVE A.H.; HOLLUND, B.E. Assessment of exposure to polycyclic aromatic hydrocarbons in engine rooms by measurement of urinary 1-hydroxypyrene. Occup. Environ. Med., v.53, p.692-696, 1996.

NIELSEN, P.S.; ANDREASSEN, A.; FARMER, P.B.; OVREBf, S.; AUTRUP, H. Biomonitoring of diesel exhaust-exposed workers. DNA and hemoglobin adducts and urinary 1-hydroxypyrene as markers of exposure. Toxicol. Lett., v.86, p.27-37, 1996.

OVREBF, S.; HAUGEN,A.; FARMER, P.B.; ANDERSON, D. Evaluation of biomarkers in plasma, blood, and urine samples from coke oven workers: significance of exposure to polycyclic aromatic hydrocarbons. Occup. Environ. Med., v.52, p.750-756, 1995.

ROGGI, C.; MONOIA, C.; SCIARRA, G.F.; APOSTOLI, P.; MACCARINI, L.; MAGNAGHI, S.; CENNI, A.; FONTE, A.; NIDASIO, G.F.; MICOLI, G. Urinary 1hydoxypyrene as a marker of exposure to pyrene: an epidemiological survey on a general population group. Sci. Total Environm., v.199, p.247-254, 1997. 
SIWINSKA, E.; MIELZYNSKA, D.; SMOLIK, E.; BUBAK, A.; KWAPULINSKI, J. Evaluation of intraand interindividual variation of urinary 1-hydroxypyrene, a biomarker of exposure to polycyclic aromatic hydrocarbons. Sci. Total Environ., v.217, p.175-183, 1998.
WORLD of HEALTH ORGANIZATION. Selected Nonheterocyclic polyciclic aromatic hydrocarbons. Geneva: WHO, 1998. 883p. (Environmental Health Criteria, 202).

Recebido para publicação em 01 de outubro de 2002. 